\title{
Percepção sobre saúde bucal e pré- natal odontológico das gestantes do município de Mineiros-GO
}

\author{
lays Fernanda OLIVEIRA'; Daiene Santos SILVA'; Daniela \\ Cristina de OLIVEIRA2; Carla Oliveira FAVRETTO²
}

1 - Discente de Graduação, Curso de Odontologia na Faculdade Morgana Potrich. Departamento de Odontopediatria. Mineiros-GO, Brasil; 2 - Doutora pelo Programa de Pós-Graduação em Ciências Odontológica, Saúde Bucal da Criança, Professora de Odontopediatria na Faculdade Morgana Potrich, Departamento de Odontopediatria. Mineiros-GO, Brasil.

\section{Resumo}

Objetivo: O objetivo deste trabalho foi avaliar o conhecimento das gestantes que fazem acompanhamento do pré-natal nas Unidades Básicas de Saúde da cidade de Mineiros-GO sobre a importância do acompanhamento do pré-natal odontológico bem como a sua percepção sobre sua saúde bucal. Material e método: Foi aplicado um questionário, contendo questões objetivas relacionadas ao nível de conhecimento de gestantes sobre o pré-natal odontológico e sua percepção sobre sua saúde bucal. Os dados foram tabulados no programa Microsoft Excel ${ }^{\circledR} 2016$ e analisados por meio de análise descritiva e quantitativa. Resultados: A idade das gestantes caracterizou um perfil jovem, com idade média de 25 anos, onde $52 \%$ apresentavam de 15 a 25 anos. Das gestantes entrevistadas, $77 \%$ consideraram sua saúde bucal boa, sendo que apenas $64 \%$ escovavam os dentes, no mínimo, 3 vezes ao dia e $76 \%$ usavam o fio dental diariamente. O pré-natal odontológico era de conhecimento de 56\% das gestantes e $44 \%$ desconheciam o programa. Das gestantes que conheciam o pré-natal odontológico, apenas $37 \%$ faziam esse acompanhamento e $33 \%$ observaram ter tido alguma alteração bucal, sendo o sangramento gengival o mais relatado. Conclusão: A maioria das gestantes são conscientes sobre a importância do pré-natal odontológico, mas nem todas fazem o acompanhamento. Além disso, apresentam um conhecimento adequado quanto aos cuidados e hábitos relacionados à saúde bucal, no entanto, algumas atitudes devem ser modificada.

PALAVRAS-CHAVE: Gestantes; Odontologia; Saúde Bucal.

\section{AUTOR PARA CORRESPONDÊNCIA}

Carla Oliveira Favretto

Departamento de Odontopediatria, Faculdade Morgana Potrich (FAMP)

Endereço: Av. Antônio Carlos Paniago, S/N, Campus II - Centro, Mineiros - G0, 75830-000

Telefone: (64) 3661-8863 / E-mail: carla.favretto@gmail.com 


\section{Introdução}

No período gestacional ocorrem várias mudanças no organismo da gestante, como alterações fisiológicas, hormonais e psicológicas, que comprometem também a cavidade oral ${ }^{1}$. Assim é indispensável que o profissional da odontologia tenha o conhecimento sobre os cuidados necessários a essa paciente, em relação a cada trimestre ${ }^{2}$.

As gestantes são um grupo de risco para doenças bucais e têm alterações fisiológicas que acarretam mudança no meio bucal, assim exigem cuidados que as tornam pacientes especiais ${ }^{3}$, por isso a importância do pré-natal odontológico, que inclui ações como orientações, controle de placa bacteriana, aplicação de flúor bem como cuidados com o futuro bebêt.

As principais alterações apresentadas pelas gestantes são o aumento de hormônios, salivação, náuseas e alterações do periodonto $^{3}$. Essas alterações associados a maus hábitos durante a gestação podem promover agravos e aparecimentos de doenças na cavidade oral como gengivite, cárie entre outras ${ }^{5}$.

O pré-natal odontológico tem como objetivo oferecer as gestantes tratamentos preventivos e curativos, além disso, este visa desmistificar crenças e mitos sobre o atendimento odontológico durante o período gestacional, no intuito de orientá-las quanto aos riscos de problemas bucais que podem desenvolver durante a gestação e da saúde oral do bebê $\hat{}^{4}$.

Todos os profissionais da saúde, principalmente da odontologia, devem atuar cada vez mais como agentes promotores de saúde, seja no contato direto ou indireto com as gestantes ${ }^{1}$. No entanto, o pré-natal odontológico ainda é negligenciado pelas gestantes, tanto pela falta de conhecimento da real importância deste acompanhamento ${ }^{6}$, quanto pelo medo de se submeterem a certos procedimentos, cabendo ao cirurgião-dentista o conhecimento e segurança em orientar e atender essa população ${ }^{7}$. 
Diante disso, o objetivo deste trabalho foi avaliar a percepção das gestantes que estavam em acompanhamento do pré-natal nas Unidades Básicas de Saúde (UBS) da cidade de MineirosGO sobre sua saúde bucal bem como conhecimento quanto à importância do pré-natal odontológico.

\section{Metodologia}

Trata-se de uma pesquisa de campo, de caráter transversal, com abordagem descritiva e quantitativa, que buscou levantar o conhecimento das gestantes atendidas nas Unidades Básicas de Saúde, na cidade de Mineiros-GO. Foram levantados dados sobre sua saúde bucal e pré-natal odontológico. Dessa forma, após a aprovação do Comitê de Ética em pesquisa da Faculdade FUNEC - Santa Fé do Sul- SP (06604919.0.0000.5428), foi aplicado um questionário com 19 questões objetivas, finalizado após a realização de um estudo piloto com todas as gestantes que estavam em acompanhamento do pré-natal e aceitaram participar do trabalho $(\mathrm{n}=100)$.

A pesquisa foi composta por uma amostra não probabilística com gestantes, de qualquer trimestre gestacional, que foram encontradas nas unidades de saúde para consulta de pré-natal e aceitaram assinar o termo de consentimento livre e esclarecido. O questionário foi aplicado na forma de entrevista na sala de recepção das unidades básicas de saúde, após o aceite dos voluntários (Quadro 1).

Os resultados foram inseridos e tabulados em um banco de dados, no programa Microsoft Excel ${ }^{\circledR} 2016$ (Microsoft Corporation), por meio da análise descritiva, cujos resultados foram expressos em forma de tabelas e gráficos.

\section{Resultados}

Para este trabalho foram entrevistadas, as gestantes $(n=100)$ que realizaram o pré-natal nas UBS na cidade de Mineiros. A idade 
QUADRO 1 - Questionário aplicado as gestantes

\section{DADOS PESSOAIS}

Nome Completo: Data de preenchimento:_________

Data de Nascimento:___________ Idade Atual____ Estado Civil: ( ) Solteira ( ) Casada ( ) Outros

Renda Familiar: ( ) Nenhuma ( ) Menos de 1 Salário mínimo ( ) Entre 1 e 2 Salários mínimos ( ) Entre 2 e 4 Salários mínimos ( ) Acima de 4 Salários mínimos

Grau de Escolaridade:
( ) Analfabeto
( ) Ensino Médio Incompleto
( ) Ensino Superior completo
( ) Ensino fundamental incompleto
( ) Ensino Médio Completo
( ) Ensino fundamental Completo
( ) Ensino Superior Incompleto

\section{SAÚDE BUCAL}

Como você considera sua saúde bucal? ( ) Ruim ( ) Boa ( ) Excelente

Quantas vezes você escova os dentes por dia, nesse período gestacional?

( ) Nenhuma ( ) 1/Uma vez ( ) 2/Duas vezes ( ) 3/três vezes ( ) 4/Quatro vezes ( ) Acima de 5/Cinco Vezes

Quantas vezes você passa o fio dental por dia, nesse período gestacional?

( ) Nenhuma ( ) 1/Uma vez ( ) 2/Duas vezes ( ) 3/três vezes ( ) 4/Quatro vezes ( ) Acima de 5/Cinco Vezes

Você já teve a doença cárie dentária?

( ) $\operatorname{Sim}$ ( ) Não ( ) Não Sei

Você controla sua alimentação de alguma forma?

( ) $\operatorname{Sim}$ ( ) Não ( ) Não Sei

Durante a gestação você procura fazer uma alimentação mais saudável?

( ) $\operatorname{Sim}$ ( ) Não ( ) Talvez

Você considera sua alimentação cariogênica?

( ) $\operatorname{Sim}$ ( ) Não ( ) Não Sei

GRAVIDEZ ATUAL

É a sua primeira gestação?

( ) $\operatorname{Sim}$ ( ) Não

Você faz acompanhamento médico, o pré natal?

( ) $\operatorname{Sim}$ ( ) Não

Você já recebeu alguma orientação do seu médico sobre a higiene bucal durante a gravidez?

( ) $\operatorname{Sim}$ ( ) Não

Você já ouviu falar do Pré Natal Odontológico?

( ) $\operatorname{sim}$

( ) Não

Se Sim, você faz esse acompanhamento?

( ) $\sin$

( ) Não

Você acredita que o acompanhamento odontológico na gestação seria importante?

( ) $\operatorname{sim}$ ( ) Não

Você procurou algum dentista durante a gestação? ( ) Sim ( ) Não

Por qual motivo? ( ) Prevenção ( ) Dor ( ) Sangramento gengival ( ) Outros

Durante a gestação você notou alguma alteração bucal? ( ) Sim （ ) Não

Se sim, qual? ( ) Presença de tártaro ( ) Sangramento da gengiva ( ) Sensibilidade dentinária

( ) Aumento focal volumétrico da gengiva (Granuloma piogênico) ( ) Outras

Fonte: Dos autores (2020) 
das gestantes caracterizou um perfil jovem, com idade média de 25 anos, onde $52 \%$ apresentavam de 15 a 25 anos, $43 \%$ de 26 a 35 anos e 5\% a idade entre 36 a 42 anos. A condição socioeconômica das entrevistadas predominante é uma renda de até quatro salários mínimos $(87 \%)$, apenas $8 \%$ possui uma renda de até 4 salários mínimos e 5\% não souberam responder. Em relação ao grau de escolaridade, a minoria apresentava ensino superior (12\%), 37\% tinham o ensino médio completo, $29 \%$ tinham o fundamental completo, $21 \%$ não concluíram o ensino fundamental e apenas $1 \%$ era analfabeta. A maioria das gestantes (67\%) são casadas e $24 \%$ solteiras (Tabela 1 ).

TABELA 1 - Distribuição das gestantes entrevistadas nas Unidades Básicas de Saúde, segundo questões socioeconômicas e educacionais (renda familiar, estado civil e escolaridade)

Características socioeconômicas

\begin{tabular}{|c|c|c|}
\hline Renda Familiar & $n$ & $\%$ \\
\hline Menos de 1 Salário mínimo & 17 & 17 \\
\hline Entre 1 e 2 Salários mínimos & 48 & 48 \\
\hline Entre 2 e 4 Salários mínimos & 22 & 22 \\
\hline Acima de 4 Salários mínimos & 8 & 8 \\
\hline \multirow[t]{2}{*}{ Em branco } & 5 & 5 \\
\hline & 100 & 100 \\
\hline Escolaridade & $n$ & $\%$ \\
\hline Analfabeto & 1 & 1 \\
\hline Ensino fundamental incompleto & 21 & 21 \\
\hline Ensino fundamental Completo & 8 & 8 \\
\hline Ensino Médio Incompleto & 21 & 21 \\
\hline Ensino Médio Completo & 28 & 28 \\
\hline Ensino Superior Incompleto & 9 & 9 \\
\hline \multirow[t]{2}{*}{ Ensino Superior completo } & 12 & 12 \\
\hline & 100 & 100 \\
\hline Estado civil & $n$ & $\%$ \\
\hline Solteira & 24 & 24 \\
\hline Casada & 67 & 67 \\
\hline \multirow[t]{2}{*}{ Outros } & 9 & 9 \\
\hline & 100 & 100 \\
\hline
\end{tabular}


Com relação a percepção das gestantes sobre sua saúde bucal, $11 \%$ das entrevistadas consideraram ter uma saúde bucal excelente, $77 \%$ boa e $12 \%$ ruim. Destas, $64 \%$ realizam a escovação diariamente, no mínimo 3 vezes ao dia e 36\% escovam 2 vezes ou menos. Sobre o uso do fio dental, 76\% usam diariamente, enquanto $24 \%$ não usam. Embora os dados apontem hábitos de higiene satisfatórios, a experiência de cárie dentária foi relatada por $81 \%$ das entrevistadas, apenas um total de $15 \%$ nunca teve a doença e 4\% não souberam responder (Tabela 2).

TABELA 2 - Conhecimento da saúde bucal das gestantes entrevistadas nas Unidades Básicas de Saúde, sobre escovação, fio dental e experiência de cárie dentária

\begin{tabular}{|c|c|c|c|}
\hline Características & Respostas & $\mathbf{n}$ & $\%$ \\
\hline \multirow{4}{*}{ Como você considera sua saúde bucal? } & Excelente & 11 & 11 \\
\hline & Boa & 77 & 77 \\
\hline & Ruim & 12 & 12 \\
\hline & Total & 100 & 100 \\
\hline \multirow{6}{*}{$\begin{array}{l}\text { Quantas vezes você escova os dentes por dia, nesse } \\
\text { período gestacional? }\end{array}$} & 1/Uma vez & 3 & 3 \\
\hline & $2 /$ Duas vezes & 33 & 33 \\
\hline & $3 /$ Três vezes & 45 & 45 \\
\hline & 4/Quatro vezes & 14 & 14 \\
\hline & Acima de $5 /$ Cinco Vezes & 5 & 5 \\
\hline & Total & 100 & 100 \\
\hline \multirow{7}{*}{$\begin{array}{l}\text { Quantas vezes você passa o fio dental por dia, nesse } \\
\text { período gestacional? }\end{array}$} & Nenhuma & 24 & 24 \\
\hline & 1/Uma vez & 32 & 32 \\
\hline & $2 /$ Duas vezes & 28 & 28 \\
\hline & 3/três vezes & 8 & 8 \\
\hline & 4/Quatro vezes & 6 & 6 \\
\hline & Acima de $5 /$ Cinco Vezes & 2 & 2 \\
\hline & Total & 100 & 100 \\
\hline \multirow{4}{*}{ Você já teve a doença cárie dentária? } & Sim & 81 & 81 \\
\hline & Não & 15 & 15 \\
\hline & Não Sei & 4 & 4 \\
\hline & Total & 100 & 100 \\
\hline
\end{tabular}


Todas as gestantes eram acompanhadas pelos médicos nas UBS de Mineiros, e apenas 54\% relataram ter recebido orientação da necessidade do cuidado bucal durante o pré-natal (Gráfico 1).

O pré-natal odontológico era de conhecimento de $56 \%$ das gestantes e $44 \%$ desconheciam o programa. Dessas gestantes que conheciam o pré-natal odontológico, apenas 37\% faziam esse acompanhamento.

No entanto, 99\% acreditam na importância de um acompanhamento odontológico, sendo que a maioria visitou um dentista nesse período $(62 \%)$, tanto na busca por prevenção (39\%) como em casos de dores (7\%) (Gráfico 2).

Em relação a doença cárie dentária, $81 \%$ das gestantes tiveram a experiência da doença e 15\% nunca apresentaram cárie. As alterações bucais foram observadas por $33 \%$ das participantes, sendo que $24 \%$ relatou ter sangramento gengival, $6 \%$ dor de dente e 3\% mau hálito (Gráfico 3).

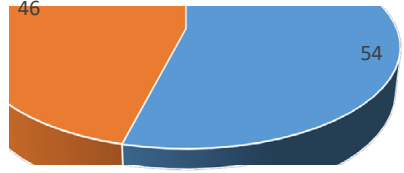

- Sim • Não
GRÁFICO 2 - Gestantes que visitaram um dentista durante a gestação e seus motivos

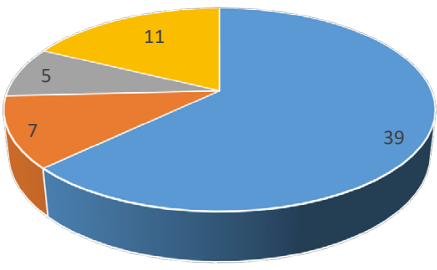

- rrevençao " vor " sangramento \# uutros

Fonte: Dados da pesquisa (2020)
GRÁFICO 3 • Principais alterações bucais observadas pelas gestantes

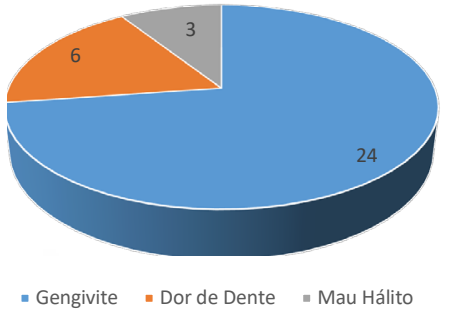

Fonte: Dados da pesquisa (2020)

\section{Discussão}

Durante a gestação, muitas vezes há a necessidade de mudanças de comportamento, como aquisição de hábitos e escolhas saudáveis, contribuindo com a promoção e manutenção de saúde do indivíduo ${ }^{3}$. Assim, se dá a importância de todo acompanhamento médico e odontológico dessa gestante. 
Neste estudo, a média de idade das gestantes foi de 25 anos, sendo que a maioria apresentava a percepção de que possuía uma boa saúde (77\%), e relatou hábitos de higienização diários. De acordo com Garbin et al. ${ }^{8}$ (2011), levando em consideração as gestantes como papel-chave dentro da família, a idade está relacionada a um fator de interferência nos cuidados com a saúde bucal. Quanto mais jovem for a gestante, menores são os cuidados não realizados em relação a sua saúde.

A percepção da importância de cuidar da saúde bucal tem aumentado nos últimos anos, em virtude do acesso as informações ${ }^{7}$. Várias UBS de Mineiros oferecem o acompanhamento através do pré-natal odontológico, contribuindo com o conhecimento apresentados pelas gestantes, corroborando com outros estudos recentes ${ }^{1}$.

$\mathrm{Na}$ amostra estudada, em relação ao grau de escolaridade, apenas $12 \%$ das gestantes apresentavam o ensino superior mostrando que a baixa escolaridade das gestantes não implica na frequência dos cuidados bucais e ainda não está relacionada com aumento de alterações bucais como relatado em alguns estudos, que a baixa escolaridade pode estar relacionada com piores condições de saúde bucal das gestantes ${ }^{9,10}$.

A cárie dentária e o aumento da incidência durante a gestação não estão relacionados às mudanças fisiológicas que acontecem nesse período, mas sim com mudanças nos hábitos de dieta e higiene bucal das gestantes ${ }^{11}$. E embora $81 \%$ das entrevistadas tenham relatado experiência passada de cárie dentária, poucas delas procuraram o dentista na época de gestação por causa de dor, devido a uma conscientização da higiene bucal.

Todas as gestantes eram acompanhadas pelos médicos nas UBS de Mineiros, e apenas 54\% relataram ter recebido orientação da necessidade do cuidado bucal durante o pré-natal, da importância de realizar as escovações diárias e sugestão de procurar um dentista para uma avaliação. De acordo com Massao ${ }^{12}$ (1996), 
o médico obstetra e o pediatra têm a importante função na prevenção de doenças bucais das gestantes, onde os mesmos devam fornecer esclarecimentos e orientações básicas em relação a hábitos de higiene bucal e dieta e, além disso, orientá-las para que vão ao cirurgião-dentista.

O pré-natal odontológico era de conhecimento de $56 \%$ das gestantes e somente $37 \%$ faziam esse acompanhamento. A condição bucal faz parte do estado de saúde que a paciente apresenta, por isso faz-se necessário a inclusão da consulta odontológica as gestantes como rotina no controle do pré-natal ${ }^{13}$. Todas as gestantes tinham direito ao acompanhamento com um dentista, mas nem todas eram cientes ou acreditavam na necessidade dessas visitas periódicas. Evidência-se assim, a necessidade da obrigatoriedade de uma consulta odontológica com o início da gestação.

No entanto, a maioria das entrevistadas acredita na importância de um acompanhamento odontológico e $62 \%$ das gestantes visitou um dentista nesse período em que a prevenção foi o principal motivo dessas consultas, reforçando a conscientização da manutenção da saúde bucal, resultados semelhantes ao encontrado por Bastiani et al. ${ }^{14}$ (2010), onde $90 \%$ das entrevistadas do estudo também relataram que seria importante o acompanhamento com o cirurgião-dentista e $46 \%$ delas visitaram um dentista durante este período por prevenção.

Estudos mostram que, durante o período gestacional, algumas alterações bucais são pertinentes, e muitas gestantes reconhecem que podem vir ocorrer problemas bucais, como a cárie e a gengivite $^{15}$. E dentre as principais alterações percebidas pelas entrevistadas, o sangramento gengival foi o mais comum (24\%), além de dor de dente, possivelmente associado a doença cárie e mau hálito. A inflamação da gengiva nessa fase deve-se às alterações periodontais que estão associadas a fatores como deficiências nutricionais, aumento de estrógeno e progesterona e presença de placa bacteriana ${ }^{16}$. 
Por fim, a necessidade desse acompanhamento é de suma importância, mas nem todas as gestantes tem esse cuidado, principalmente pela falha na inserção do cirurgião-dentista nos serviços de pré-natal, pelo despreparo no assunto e a omissão por parte dos profissionais de saúde, além do receio das gestantes de irem ao dentista ${ }^{14}$.

\section{Conclusão}

Conclui-se com este trabalho, que a maioria das gestantes são conscientes sobre a importância do pré-natal odontológico, mas nem todas fazem o acompanhamento. Além disso, apresentam um conhecimento adequado quanto aos cuidados e hábitos relacionados à saúde bucal, no entanto, algumas atitudes devem ser modificadas.

Além disso, o pré-natal odontológico deve ser oferecido a todas, como forma de levar ao conhecimento sobre os cuidados bucais bem como tratar precocemente qualquer alteração bucal, minimizando problemas que venham implicar com o bem-estar tanto da mãe como do bebê.

\section{Referências}

1 - Zemolin AB, Sassi ET, Willms GP, Rech JP, Dalsochio L. Gestantes: da saúde bucal a saúde geral. Rev Saúde Integr. 2017; 10(20): 76-80.

2- Vasconcelos RG, Vasconcelos MG, Mafra RP, Alves Júnior LC, Queiroz LMG, Barboza CAG. Atendimento odontológico a pacientes gestantes: como proceder com segurança. Rev Bras Odontol. 2012; 69(1): 120-4.

3 - Nascimento EP, Andrade FS, Costa AMDD, Terra FS. Gestantes frente ao tratamento odontológico. Rev Bras Odontol. 2012; 69(1): 125-30.

4 - Konishi F, Abreu-e-Lima F. Odontologia intra-uterina: a construção da saúde bucal antes do nascimento. Rev Bras Odont. 2002; 59(5): 294-5.

5 - Rossel FL. Prevalência de fatores clínicos do risco de cárie em gestantes. [Tese de Doutorado]. Araraquara: Faculdade de Odontologia da Universidade Estadual Paulista "Júlio de Mesquita Filho"; 1998. 95 p.

6 - Massoni ACLT, Pereira RB, Nóbrega DRM, Costa LED, Fernandes JMFA, Rosenblatt A. Avaliação do conhecimento de gestantes e puérperas primíparas e multíparas sobre cárie dentária. Rev Gaúch Odontol. 2015; 63(2): 145-52. 
7 - Santos Neto ET, Oliveira AE, Zandonade E, Leal MC. Acesso à assistência odontológica no acompanhamento pré-natal. Ciênc Saúde Colet. 2012; 17(11): 3057-68.

8 - Garbin CAS, Sumida DH, Santos RR, Chehoud KA, Moimaz SAS. Saúde coletiva: promoção de saúde bucal na gravidez. Rev Odontol UNESP. 2011; 40(4): 161-5

9 - Coimbra LC, Silva MAS, Mochel EGM, Alves MTSSB, Ribeiro VS, Aragão VMF, et al. Fatores associados à inadequação do uso da assistência pré-natal. Rev Saúde Pública. 2003; 37(4): 456-62.

10 - Leal MC, Gama SGN, Cunha CB. Desigualdades raciais, sócio-demográficas e na assistência ao pré-natal e ao parto. Rev Saúde Pública. 2005; 39(1): 100-7.

11 - Miller MC. The pregnant dental patient. J Can Dent Assoc. 1995; 23: 63-70.

12 - Massao JM, Sued ML, Giordano DV, Gama RS, Santos RA, Carneiro AA. Filosofia da clínica de bebês da UNIGRANRIO- RJ. Rev Bras Odont. 1996; 53(5): 6-13.

13 - Feldens EG, Feldens CA, Kramer PF, Class BM, Marco CC. A Percepção dos médicos obstetras a respeito da saúde bucal da gestante. Pesq Bras Odontop Clin Integr. 2005; 5(1): 41-6.

14 - Bastiani C, Cota ALS, Provenzano MGA, Fracasso MLC, Honório HM, Rios D. Conhecimento das gestantes sobre alterações bucais e tratamento odontológico durante a gravidez. Rev Odontol Clín Cient. 2010; 9(2) 155-60.

15 - Martins RFO, Martins ZIO. O que as gestantes sabem sobre cárie: uma avaliação dos conhecimentos de primigestas e multigestas quanto à própria saúde bucal. Rev ABO Nac. 2002; 10(2): 278-84.

16 - Romero RD, Sanches CM. Los odontologos educan a sus pacientes. Rev ADM. 1988; 55(2): 317-20. 


\title{
Pregnant women's perception of oral health and prenatal dentistry in Mineiros
}

\begin{abstract}
Objective: The objective of this study was to evaluate the knowledge of pregnant women who follow prenatal care in Basic Health Units in the city of Mineiros-GO about the importance of monitoring dental prenatal care as well as their perception of their oral health. Material and method: A questionnaire was applied, containing objective questions related to the level of knowledge of pregnant women about dental prenatal care and their perception of their oral health. The data were tabulated in the Microsoft Excel ${ }^{\circledR} 2016$ program and analyzed using descriptive and quantitative analysis. Results: The age of the pregnant women characterized a young profile, with an average age of 25 years old, that $52 \%$ were between 15 and 25 years old. Of the pregnant women interviewed, $77 \%$ considered their oral health to be good, with only $64 \%$ brushing their teeth at least 3 times a day and $76 \%$ using dental floss daily. Dental prenatal care was known to $56 \%$ of pregnant women and $44 \%$ unaware the program. Of the pregnant women who knew about dental prenatal care, only $37 \%$ underwent this follow-up and $33 \%$ observed having had some oral alteration, with gum bleeding being the most reported. Conclusion: Most pregnant women are aware of the importance of dental prenatal care, but not all follow up. Furthermore, they have adequate knowledge about the care and habits related to oral health, however, some attitudes must be modified.
\end{abstract}

KEYWORDS: Pregnant women; Dentistry; Oral Health. 